\title{
Neutron scattering studies on short- and long-range layer structures and related dynamics in imidazolium-based ionic liquids
}

Cite as: J. Chem. Phys. 149, 054502 (2018); https://doi.org/10.1063/1.5037217

Submitted: 22 April 2018 . Accepted: 05 July 2018 . Published Online: 02 August 2018

Fumiya Nemoto, Maiko Kofu (D), Michihiro Nagao, Kazuki Ohishi, Shin-ichi Takata, Jun-ichi Suzuki, Takeshi Yamada, Kaoru Shibata, Takeshi Ueki, Yuzo Kitazawa, Masayoshi Watanabe, and Osamu Yamamuro

\section{ARTICLES YOU MAY BE INTERESTED IN}

Dynamic and structural evidence of mesoscopic aggregation in phosphonium ionic liquids The Journal of Chemical Physics 148, 193815 (2018); https://doi.org/10.1063/1.5009765

Quasielastic neutron scattering studies on glass-forming ionic liquids with imidazolium cations

The Journal of Chemical Physics 143, 234502 (2015); https://doi.org/10.1063/1.4937413

Preface: Special Topic on Chemical Physics of Ionic Liquids

The Journal of Chemical Physics 148, 193501 (2018); https://doi.org/10.1063/1.5039492

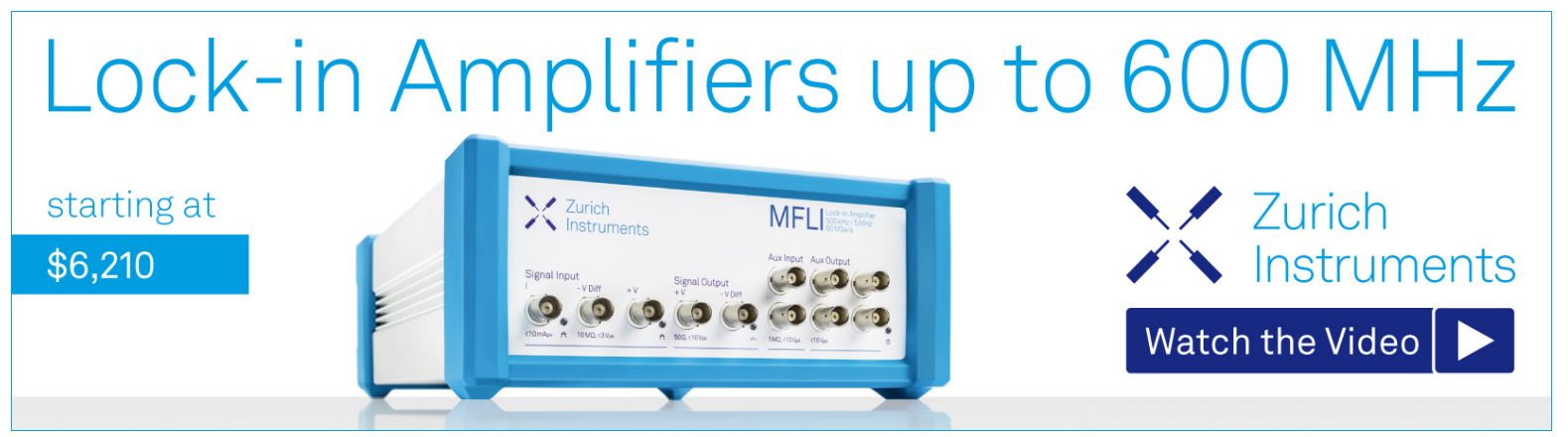

J. Chem. Phys. 149, 054502 (2018); https://doi.org/10.1063/1.5037217 


\title{
Neutron scattering studies on short- and long-range layer structures and related dynamics in imidazolium-based ionic liquids
}

\author{
Fumiya Nemoto, ${ }^{1, a)}$ Maiko Kofu, ${ }^{1,2}$ Michihiro Nagao, ${ }^{3,4}$ Kazuki Ohishi, ${ }^{5}$ Shin-ichi Takata, ${ }^{2}$ \\ Jun-ichi Suzuki, ${ }^{5}$ Takeshi Yamada, ${ }^{5}$ Kaoru Shibata, ${ }^{2}$ Takeshi Ueki, ${ }^{6, b)}$ Yuzo Kitazawa, ${ }^{7}$ \\ Masayoshi Watanabe, ${ }^{7}$ and Osamu Yamamuro, c) \\ ${ }^{1}$ Institute for Solid State Physics, University of Tokyo, 5-1-5 Kashiwanoha, Kashiwa, Chiba 277-8581, Japan \\ ${ }^{2} J$-PARC Center, Japan Atomic Energy Agency, 2-4 Shirakata, Tokai, Naka, Ibaraki 319-1195, Japan \\ ${ }^{3}$ NIST Center for Neutron Research, National Institute of Standards and Technology, 100 Bureau Drive, \\ Gaithersburg, Maryland 20899-6102, USA \\ ${ }^{4}$ Center for Exploration of Energy and Matter, Indiana University, Bloomington, Indiana 47408-1398, USA \\ ${ }^{5}$ Neutron Science and Technology Center, Comprehensive Research Organization for Science and Society \\ (CROSS), IQBRC Bldg., 162-1 Shirakata, Tokai, Naka, Ibaraki 319-1106, Japan \\ ${ }^{6}$ Department of Materials Engineering, University of Tokyo, 7-3-1 Hongo, Bunkyo, Tokyo 113-8656, Japan \\ ${ }^{7}$ Department of Chemistry and Biotechnology, Yokohama National University, 79-5 Tokiwadai, Hodogaya, \\ Yokohama, Kanagawa 240-8501, Japan
}

(Received 22 April 2018; accepted 5 July 2018; published online 2 August 2018)

\begin{abstract}
Alkyl-methyl-imidazolium ionic liquids $\mathrm{C} n \operatorname{mimX}$ ( $n$ : alkyl-carbon number, $\mathrm{X}$ : anion) have short-range layer structures consisting of ionic and neutral (alkylchain) domains. To investigate the temperature dependences of the interlayer, interionic group, and inter-alkylchain correlations, we have measured

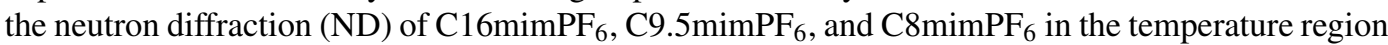
from $4 \mathrm{~K}$ to $470 \mathrm{~K}$. The quasielastic neutron scattering (QENS) of $\mathrm{C}_{16} \mathrm{mimPF}_{6}$ was also measured to study the dynamics of each correlation. $\mathrm{C}_{16 \mathrm{mimPF}_{6}}$ shows a first-order transition between the liquid (L) and liquid crystalline (LC) phases at $T_{\mathrm{c}}=394 \mathrm{~K}$. C8mimPF $\mathrm{m}_{6}$ exhibits a glass transition at $T_{\mathrm{g}}=200 \mathrm{~K}$. C9.5 $\mathrm{mimPF}_{6}$, which is a $1: 3$ mixture between $\mathrm{C} \mathrm{mimPF}_{6}$ and $\mathrm{C} 10 \mathrm{mimPF}_{6}$, has both transitions at $T_{\mathrm{c}}=225 \mathrm{~K}$ and $T_{\mathrm{g}}=203 \mathrm{~K}$. In the ND experiments, all samples exhibit three peaks corresponding to the correlations mentioned above. The widths of the interlayer peak at ca. $0.2 \AA^{-1}$ changed drastically at the L-LC transitions, while the interionic peaks at ca. $1 \AA^{-1}$ exhibited a small jump at $T_{\mathrm{c}}$. The peak position and area of the three peaks did not change much at the transition. The structural changes were minimal at $T_{\mathrm{g}}$. The QENS experiments demonstrated that the relaxation time of the interlayer motion increased tenfold at $T_{\mathrm{c}}$, while those of other motions were monotonous in the whole temperature region. The structural and dynamical changes mentioned above are characteristic of the L-LC transition in imidazolium-based ionic liquids. Published by AIP Publishing. https://doi.org/10.1063/1.5037217
\end{abstract}

\section{INTRODUCTION}

Ionic liquid (IL) is one of the most attractive materials in current liquid science. ${ }^{1-3}$ ILs have many remarkable properties suitable for the practical applications, e.g., low melting temperature, negligible vapor pressure, non-flammability, high ionic conductivity, amphiphilicity, high designability, and so on. In fact, ILs are applied as green solvents, ${ }^{1}$ electrochemical materials, ${ }^{2}$ actuators, ${ }^{2}$ and lubricants. ${ }^{3}$ Among ILs, the imidazolium-based ones including our target materials 1alkyl-3-methylimidazolium salts are studied most intensively. They are usually abbreviated as $\mathrm{CnmimX}$ where $n$ is the alkyl-carbon number and $\mathrm{X}$ is the anion.

\footnotetext{
a)Present address: Institute of Materials Structure Science/J-PARC Center, High Energy Accelerator Research Organization (KEK), 203-1 Shirakata, Tokai, Naka, Ibaraki 319-1106, Japan.

b)Present address: International Center for Materials Nanoarchitectonics, National Institute for Materials Science, 1-1 Namiki, Tsukuba, Ibaraki 305-0044, Japan.

c)Electronic mail: yamamuro@ issp.u-tokyo.ac.jp
}

During the past decade, great progress has also been made in fundamental scientific studies. ${ }^{4-6}$ The structural investigations for $\mathrm{C} n \operatorname{mimX}$ with $n<10$ have been performed by using $\mathrm{x}$-ray and neutron diffraction (ND) techniques ${ }^{7-21}$ and molecular dynamics (MD) simulations. ${ }^{12,17,22-30}$ In these studies, a characteristic peak indicative of $Q_{\text {low }}$, which is called "low- $Q$ peak" in this paper, appeared at $Q_{\text {low }} \approx 0.3 \AA^{-1}$ in addition to the peaks at $Q_{\text {ion }} \approx 1 \AA^{-1}$ corresponding to the correlation among ions and at $Q_{\text {alkyl }} \approx 1.5 \AA^{-1}$ corresponding mainly to the correlation among alkylchains (Fig. 1) ${ }^{8-21}$ These studies indicate that $\mathrm{CnmimX}$ have some higher-order structures in nanometer scale, which we call "nanostructure." The nanostructure consists of the aggregations of the charged parts of cations and anions and the neutral parts of alkylchains. On the other hand, CnmimX with $n \geq 14$ have smectic A (SmA) liquid-crystalline (LC) phases as a lower temperature phase of the liquid (L) phase. ${ }^{31-45}$ The structures of the LC phases were investigated by both $\mathrm{x}$-ray diffraction experiments ${ }^{32-39}$ and MD simulations. ${ }^{40-45}$ Our thermal and x-ray diffraction work ${ }^{39}$ has shown that the nanostructure of CnmimX with 

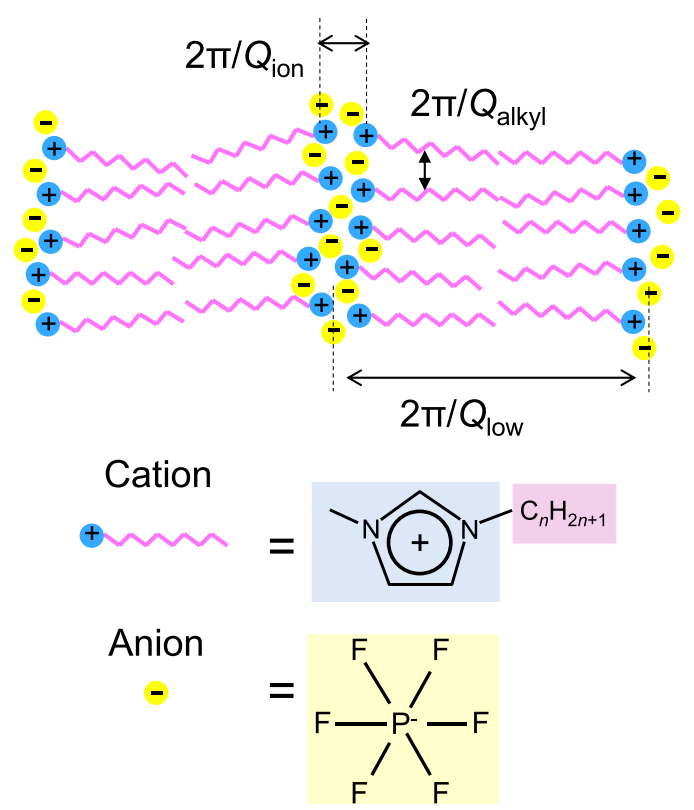

FIG. 1. Schematic drawing of the layer structure and the chemical structure of 1-alkyl-3-methylimidazolium hexafluorophosphate $\left(\mathrm{CnmimPF}_{6}\right.$; $n$ is alkylcarbon number). $Q_{\text {ion }}, Q_{\text {alkyl }}$, and $Q_{\text {low }}$ are the positions of the three characteristic peaks commonly observed in the diffraction patterns of $\mathrm{CnmimPF}_{6}$.

$n<14$ is the short-range ordered form of the layer structure of the SmA LC phase.

The dynamics of CnmimX are also investigated mainly in the context of the non-Stokes-Einstein relation which makes a connection between the microscopic and macroscopic dynamics. Microscopic motions have been investigated by nuclear magnetic resonance (NMR), ${ }^{46-51}$ quasielastic neutron scattering (QENS), and inelastic x-ray scattering measurements, $8,13,15,52-63$ while macroscopically by viscoelastic, ${ }^{16,46,47,55,58,62,64-71}$ dielectric, ${ }^{49,50,55,71-77}$ and ionic conductivity measurements. $46,47,68,70,71,78-80$ Among these measurement methods, QENS is especially suitable for the research of ILs since either coherent or incoherent scattering can be chosen by selective deuteration. This feature is based on the characteristic scattering cross sections of neutrons $\sigma$; i.e., $\sigma_{\text {coh }}(H)=1.76$ barn, $\sigma_{\text {inc }}(H)=80.27$ barn, $\sigma_{\text {coh }}(D)=5.59$ barn, and $\sigma_{\text {inc }}(D)=2.05$ barn. The coherent neutron scattering gives information about a specific collective motion with a finite length scale by choosing a scattering vector $Q$. On the other hand, the incoherent neutron scattering reflects the self-motions. Our coherent neutron spin echo (NSE) measurements on fully deuterated ILs, d-C 8 mimPF $_{6}$ and d-C8mimTFSI [TFSI: bis(trifluoromethylsulfonyl)imide], revealed the relaxation of the nanostructure with relaxation times $\tau$ of $10 \mathrm{~ns}-100 \mathrm{~ns}$ and a large activation energy $\Delta E_{\mathrm{a}}$ of ca. $40 \mathrm{~kJ} \mathrm{~mol}^{-1} .{ }^{15}$ The several incoherent QENS studies ${ }^{8,52-54,56,57,59,61,62}$ on hydrogenated CnmimX $(n<10)$ presented the relaxation of the alkylchains with $\tau$ of the order of ps and a small $\Delta E_{\mathrm{a}}$ (ca. $5 \mathrm{~kJ} \mathrm{~mol}^{-1}$ ) and that of the ionic diffusion with intermediate $\tau$ and $\Delta E_{\mathrm{a}}$. For $\mathrm{C}_{16} \mathrm{mimPF}_{6}$ with an LC phase, Triolo et al. ${ }^{36}$ reported that the incoherent elastic intensity does not change at the LC-L transition on the neutron spectroscopy with a $1 \mu \mathrm{eV}$ energy resolution, suggesting that any motional transition if any may appear at a $\tau$ slower than $1 \mathrm{~ns}$.

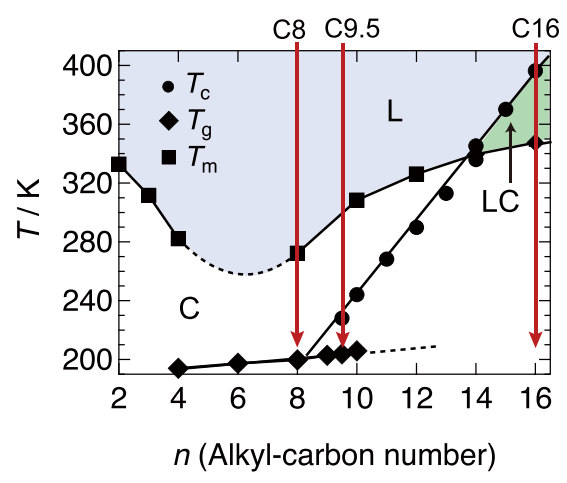

FIG. 2. Alkyl-carbon number $(n)$ dependences of the transition temperatures in $\mathrm{CnmimPF}_{6} . T_{\mathrm{c}}$ is the transition temperature between the liquid crystal (LC) and liquid (L), $T_{\mathrm{g}}$ is the glass transition temperature, and $T_{\mathrm{m}}$ is the melting temperature of the crystalline $(\mathrm{C})$ phase ${ }^{39}$ In this work, $\mathrm{C} 8 \mathrm{mimPF}_{6}, \mathrm{C} 9.5 \mathrm{mimPF}_{6}$

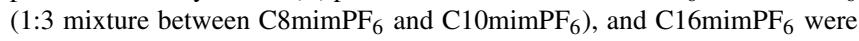
investigated. The error bars are smaller than the symbols.

The main purpose of this study is to clarify the temperature dependence of the nanostructure and related dynamics, especially changes at the LC-L transition. The ionic and alkylchain correlations with a smaller spatial scale and a shorter time scale are also within our scope. Hence we have used diffractometers with a wide $Q$-range $\left(5 \times 10^{-3} \AA^{-1}<Q<17 \AA^{-1}\right)$ and two QENS spectrometers covering a wide time range $(1 \mathrm{ps}<t<40 \mathrm{~ns})$. The measured samples are $\mathrm{h}-$ and $\mathrm{d}-$ $\mathrm{C}_{16} \mathrm{mimPF}_{6}$ having an $\mathrm{LC}-\mathrm{L}$ transition, $\mathrm{d}-\mathrm{C} 8 \mathrm{mimPF}_{6}$ showing a glass transition, and $\mathrm{d}-\mathrm{C} 9.5 \mathrm{mimPF}_{6}$ exhibiting both transitions; $\mathrm{C} .5 \mathrm{mimPF}_{6}$ is not a pure IL but a $1: 3$ mixture of $\mathrm{C} \mathrm{mimPF}_{6}$ and $\mathrm{C}_{10 \mathrm{mimPF}}$. The $\mathrm{C} 9.5 \mathrm{mimPF}_{6}$ sample is considered to be homogeneous from the smoothness of the transition lines in the phase diagram, the sharpness of the transition peaks in the differential scanning calorimetry (DSC) data, and the unity of the low- $Q$ peak in the x-ray diffraction pattern. ${ }^{39}$ The d-samples were used for diffraction and coherent QENS experiments and h-C16mimPF 6 for incoherent QENS to investigate the ionic and alkyl motions. The phase relations of the three samples are shown in the $n-T$ phase diagram determined previously (Fig. 2). ${ }^{39}$

\section{EXPERIMENTAL SECTION}

\section{A. Samples and differential scanning calorimetry}

Totally protonated $\mathrm{C}_{16} \mathrm{mimPF}_{6}\left(\mathrm{~h}-\mathrm{C} 16 \mathrm{mimPF}_{6}\right)$, whose purity was claimed to be above $98 \%$, was purchased from Iolitec, Inc., and used without further purification.

The starting materials for the syntheses of the deuterated samples 1-methylimidazole- $\mathrm{d}_{6}(98 \%$ atom D), 1bromohexadecane- $\mathrm{d}_{33}$ (98\% atom D), 1-bromodecane- $\mathrm{d}_{21}$ $(98 \%$ atom $\mathrm{D})$, and 1-bromooctane- $\mathrm{d}_{17}(98 \%$ atom $\mathrm{D})$ were purchased from $\mathrm{C} / \mathrm{D} / \mathrm{N}$ isotope, Inc., and $\mathrm{D}_{2} \mathrm{O}, \mathrm{CDCl}_{3}$, and $\mathrm{NaPF}_{6}$ from Sigma-Aldrich Corp. These chemical reagents were used without purifications. Fully deuterated samples d-C16mimPF 6 , d-C10mimPF 6 , and d-C8mimPF 6 were prepared by using the procedure reported earlier. ${ }^{15}$ In order to remove the remaining (unreacted) chemical reagents used in the syntheses and moisture, $\mathrm{d}-\mathrm{C} 16 \mathrm{mimPF}_{6}$ was dried under vacuum for $22 \mathrm{~h}$ at $423 \mathrm{~K}$ and d-C $8 \mathrm{mimPF}_{6}$ and d-C $10 \mathrm{mimPF}_{6}$ 
for $10 \mathrm{~h}$ at $403 \mathrm{~K}$. d-C $9.5 \mathrm{mimPF}_{6}$ was prepared by mixing d-C $8 \operatorname{mimPF}_{6}$ and d-C10mimPF 6 at a mole ratio of $1: 3$ using a gravimetric method. This mixing method is useful for suppressing crystallization and enables us to observe both the glass and LC-L transition as described later.

We have performed differential scanning calorimetry (DSC) of the above samples using PerkinElmer Diamond DSC mainly to check the purities of the deuterated samples. The scanning rate was $5 \mathrm{~K} \mathrm{~min}^{-1}$ for all runs. The DSC charts demonstrated step-like anomalies due to the glass transition and sharp peaks due to the melting and the LC-L transition. No extra peak from impurities was observed. All of the transition temperatures are summarized in Table I. As shown in this table, the transition temperatures of the deuterated samples are close to those of the hydrogenated samples, ${ }^{39}$ indicating good purities of the deuterated samples. The transition temperatures in Table I are useful also to determine the temperature ranges for the neutron scattering measurements.

\section{B. Wide $Q$-range neutron diffraction}

The neutron diffraction data of d-C16mimPF${ }_{6}$, d-C9.5 $\mathrm{mimPF}_{6}$, and $\mathrm{d}-\mathrm{C} 8 \mathrm{mimPF}_{6}$ were collected using BL15 Small and Wide Angle Neutron Scattering Instrument TAIKAN $^{81}$ at the Material and Life Science Experimental Facility (MLF), Japan Proton Accelerator Research Complex (J-PARC). TAIKAN is a time-of-flight (TOF) instrument using pulsed neutrons with a wide wavelength range $(0.5 \AA-$ $8 \AA$ ), providing high quality of small-angle neutron scattering (SANS) and neutron diffraction (ND) data in a wide $Q$ range $\left(5 \times 10^{-3} \AA^{-1}-17 \AA^{-1}\right)$. This wide $Q$-range is suitable for complex materials with higher-order structures such as ILs. In the present work, each sample was fused in an aluminum cylindrical cell with an inner diameter of $6 \mathrm{~mm}$. This sample geometry and high transmission of neutrons reduce the strong alignment effect, which was a serious problem in our XRD measurement with reflective optics. ${ }^{39}$ The sample temperature was controlled by our custom-built high temperature stage $(300 \mathrm{~K}-470 \mathrm{~K})$ for $\mathrm{d}-\mathrm{C} 16 \mathrm{mimPF}_{6}$ and by a standard bottom-loading cryostat $(4 \mathrm{~K}-300 \mathrm{~K})$ of TAIKAN for $\mathrm{d}-\mathrm{C} 8 \mathrm{mimPF}_{6}$ and $\mathrm{d}-\mathrm{C} 9.5 \mathrm{mimPF}_{6}$. The SANS and ND data were processed by the Utsusemi software ${ }^{82}$ developed by J-PARC.

\section{Small angle neutron scattering}

Small angle neutron scattering (SANS) measurements for $\mathrm{d}_{-} \mathrm{C} 6 \mathrm{mimPF}_{6}$ near $T_{\mathrm{c}}$ were performed using

TABLE I. Transition temperatures for the samples measured in this work. $T_{\mathrm{m}}$ is the melting temperature, $T_{\mathrm{c}}$ is the $\mathrm{LC}-\mathrm{L}$ transition temperature, and $T_{\mathrm{g}}$ is the glass transition temperature. These temperatures were determined with DSC; the uncertainty is smaller than $\pm 0.5 \mathrm{~K}$ for $T_{\mathrm{m}}$ and $T_{\mathrm{c}}$ and $\pm 1 \mathrm{~K}$ for $T_{\mathrm{g}}$ as is claimed for standard DSC instruments.

\begin{tabular}{|c|c|c|c|c|c|c|}
\hline & \multicolumn{2}{|c|}{$\mathrm{C} \mathrm{mimPF}_{6}$} & \multicolumn{2}{|c|}{$\mathrm{C} 9.5 \mathrm{mimPF}_{6}$} & \multicolumn{2}{|c|}{$\mathrm{C}_{16 \mathrm{mimPF}_{6}}$} \\
\hline & $\mathrm{h}^{39}$ & $\mathrm{~d}$ & $\mathrm{~h}^{39}$ & $\mathrm{~d}$ & $h^{39}$ & $\mathrm{~d}$ \\
\hline$T_{\mathrm{m}}(\mathrm{K})$ & 272.3 & 268.6 & $\ldots$ & $\ldots$ & 347.5 & 343.7 \\
\hline$T_{\mathrm{c}}(\mathrm{K})$ & $\ldots$ & $\ldots$ & 228.1 & 225.1 & 396.3 & 394.3 \\
\hline$T_{\mathrm{g}}(\mathrm{K})$ & 200 & 200 & 204 & 202 & $\cdots$ & \\
\hline
\end{tabular}

NGB-30m-SANS ${ }^{83}$ at the NIST Center for Neutron Research (NCNR), National Institute of Standards and Technology (NIST), USA. The average wavelength of used neutrons was $5 \AA$ with the wavelength spread of about $12.5 \%$, and data were collected at a $Q$ range of $0.03 \AA^{-1}-0.35 \AA^{-1}$. The sample was loaded in an annular hollow cylindrical cell made of aluminum. The sample cell has an outer diameter of $18 \mathrm{~mm}$ and a gap (sample thickness) of $2 \mathrm{~mm}$. The sample temperature was controlled with a closed cycle refrigerator $(10 \mathrm{~K}-440 \mathrm{~K})$. The SANS data were processed by a software package ${ }^{84}$ based on IGOR Pro developed at NCNR.

\section{Backscattering instrument for QENS}

QENS data of h-C16mimPF 6 and d-C16mimPF 6 were collected using BL02 TOF near-backscattering spectrometer DNA ${ }^{85}$ at MLF, J-PARC. The neutrons scattered from a sample were energy-analyzed at $2.02 \mathrm{meV}$ by means of $\mathrm{Si}$ (111) analyzers with a scattering Bragg angle $\theta_{\mathrm{B}}$ of $87.5^{\circ}$. We used the following two resolution modes switched by a pulseshaping chopper-high resolution mode: energy resolution $\Delta E$ $=3.6 \mu \mathrm{eV}$, energy window $-40 \mu \mathrm{eV}<E<100 \mu \mathrm{eV}$, momentum transfer range $0.13 \AA^{-1}<Q<1.88 \AA^{-1}$ and low resolution mode: $\Delta E=14 \mu \mathrm{eV},-500 \mu \mathrm{eV}<E<1500 \mu \mathrm{eV}, 0.13 \AA^{-1}$ $<Q<1.98 \AA^{-1}$.

The samples were loaded in an annular hollow cylindrical cell made of aluminum. The sample cell has an outer diameter of $18 \mathrm{~mm}$ and the gap (sample thickness) of $0.3 \mathrm{~mm}$ for h-C16mimPF 6 and $2 \mathrm{~mm}$ for $\mathrm{d}-\mathrm{C}_{16} \mathrm{mimPF}_{6}$. The resolution functions $R(Q, \omega)$ were measured at $10 \mathrm{~K}$ where no relaxation (no QENS) was observed. The QENS spectra were corrected by the background (empty cell) data measured separately. The sample temperature was controlled by a standard top-loading cryostat installed to DNA $(10 \mathrm{~K}-450 \mathrm{~K})$. The QENS data were processed by the Utsusemi software, and the fitting of the QENS spectra was performed using the DAVE software package $^{86}$ developed at NCNR.

\section{E. NSE}

The NSE measurement of d-C16mimPF 6 was performed using NGA NSE ${ }^{87}$ at NCNR, NIST, USA. The wavelengths of used neutrons were $6 \AA$ and $8 \AA$ with the wavelength resolution of about $20 \%$. The spin echo measurements were carried out at $Q_{\text {low }}=0.2 \AA^{-1}$. The data were collected at Fourier times between $0.006 \mathrm{~ns}$ and $40 \mathrm{~ns}$. The sample cell was identical to the one used in the SANS and DNA measurements. The resolution function was measured by using charcoal powder at room temperature. The sample temperature was controlled with a standard closed cycle refrigerator $(350 \mathrm{~K}-473 \mathrm{~K})$ of NCNR. The raw echo data were corrected with the empty cell data and processed to the intermediate scattering functions by using the DAVE software package.

\section{RESULTS AND DISCUSSION}

\section{A. Neutron diffraction}

\section{Overall feature of structure}

Figure 3 shows the diffraction patterns of the L (420 K), $\mathrm{LC}(350 \mathrm{~K})$, and crystalline $(300 \mathrm{~K})$ phases of d-C16 $\mathrm{mimPF}_{6}$ 


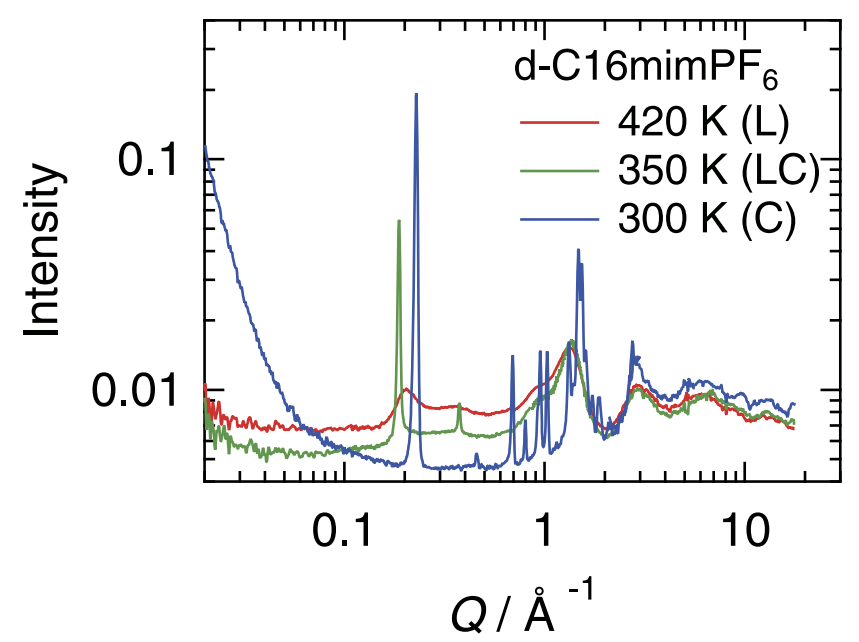

FIG. 3. Overall features of the neutron diffraction patterns for liquid (L), liquid crystalline (LC), and crystalline (C) phases of d-C16mimPF $\mathrm{P}_{6}$ obtained by TAIKAN.

obtained by TAIKAN. In the low $Q$ region $\left(Q<0.1 \AA^{-1}\right)$, a strong scattering proportional to $Q^{-4}$ was observed only in the crystalline (C) phase. This is attributed to the scattering from the grain boundaries which were generated at crystallization. For the $\mathrm{L}$ and $\mathrm{LC}$ phases, the absence of the scattering in the low $Q$ region indicates that there is no domain-like structure larger than the length scale (ca. $30 \AA$ ) corresponding to the low- $Q$ peak. In the medium $Q$ region $\left(0.1 \AA^{-1}<Q<2 \AA^{-1}\right)$, the difference among the three phases is vivid. In the $\mathrm{L}$ phase, as described in the Introduction, the three broad peaks corresponding to the inter-layer (low- $Q$ ), inter-ionic, and interalkylchain correlations appeared at around $Q_{\text {low }} \approx 0.2 \AA^{-1}$, $Q_{\text {ion }} \approx 1.0 \AA^{-1}$, and $Q_{\text {alkyl }} \approx 1.5 \AA^{-1}$, respectively. In the LC phase, the low- $Q\left(Q_{\text {low }}\right)$ peak and its higher-order one became much sharper, while the inter-ionic $\left(Q_{\text {ion }}\right)$ and inter-alkylchain $\left(Q_{\text {alkyl }}\right)$ peaks are almost unchanged from the $\mathrm{L}$ phase. In the $\mathrm{C}$ phase, there are many sharp Bragg peaks as usual. In the higher $Q$ region $\left(Q>2 \AA^{-1}\right)$, the diffraction curves of the three phases are quite similar although the intensity of the $C$ phase is slightly larger. This is not surprising because the broad component at a higher $Q$ region reflects the intra-molecular or intra-ionic structure. The slightly larger intensity of the $\mathrm{C}$ phase may be due to the superposition of many small Bragg peaks and/or the increase of density in the $\mathrm{C}$ phase.

\section{Temperature dependence of diffraction peaks}

a. Comparison among the three samples. Figure 4 shows the diffraction patterns (in the medium $Q$ region) of d-C16mimPF 6 , d-C8mimPF 6 , and d-C9.5mimPF 6 observed by TAIKAN at several temperatures. The shape of the low- $Q$ peak of d-C $16 \mathrm{mimPF}_{6}$ and d-C9.5 $\mathrm{mimPF}_{6}$ changed drastically at $T_{\mathrm{c}}$ of the LC-L transition. On the other hand, the low- $Q$ peak of d-C $8 \mathrm{mimPF}_{6}$ was continuous because of the lack of the LC$\mathrm{L}$ transition, but its intensity increased steeply with decreasing temperature. The shorter the alkylchain length is, the higher the position of the low- $Q$ peak $\left(Q_{\text {low }}\right)$ becomes. This is consistent with the fact that $Q_{\text {low }}$ corresponds to the distance between the ionic layer separated by alkylchains (see Fig. 1). On the other hand, the positions of the ionic peak $\left(Q_{\text {ion }}\right)$ and alkyl peak

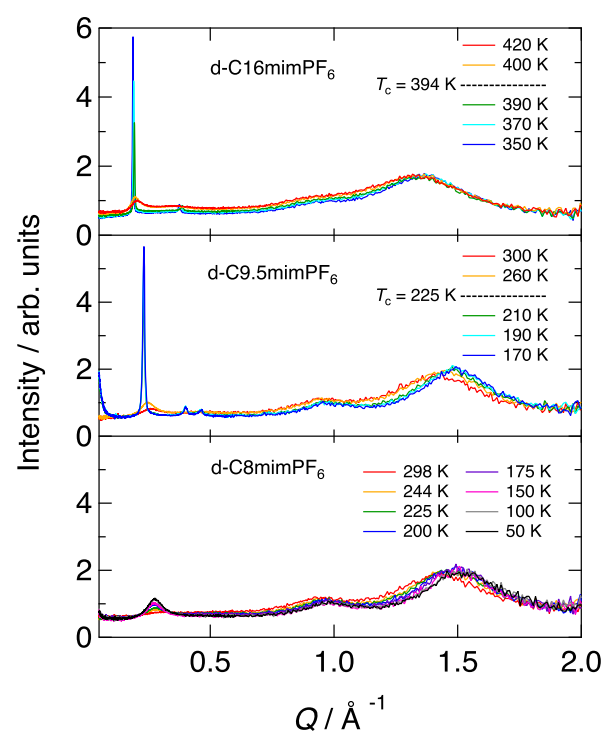

FIG. 4. Neutron diffraction patterns in the medium- $Q$ range at several temperatures for $\mathrm{d}_{-} \mathrm{C} 8 \mathrm{mimPF}_{6}, \mathrm{~d}-\mathrm{C} 9.5 \mathrm{mimPF}_{6}$, and $\mathrm{d}-\mathrm{C} 16 \mathrm{mimPF}_{6}$ obtained by TAIKAN.

$\left(Q_{\text {alkyl }}\right)$ were almost independent of the alkylchain length in the comparison at the same temperature, e.g., $Q_{\text {ion }}=0.95 \AA^{-1}$ and $Q_{\text {alkyl }}=1.4 \AA^{-1}$ at $300 \mathrm{~K}$ (extrapolation was needed for d-C16mimPF ${ }_{6}$ ). This suggests that the structure of the ionic part remains unchanged with the alkylchain length. The result is in agreement with the micro phase separation picture of the nanostructure. In $\mathrm{d}-\mathrm{C} 9.5 \mathrm{mimPF}_{6}$, a small peak appeared at $0.45 \AA^{-1}$ in addition to the second-order peak of low- $Q$ peaks at $0.4 \AA^{-1}$. This peak is attributed to neither a higher-order peak of the low- $Q$ peak nor a Bragg peak in the $\mathrm{C}$ phase. It is possible that this unknown peak is due to some unexpected inhomogeneity caused by mixing d-C $8 \mathrm{mimPF}_{6}$ and $\mathrm{d}-\mathrm{C} 10 \mathrm{mimPF}_{6}$. Actually, a small angle scattering, which could be due to some phase separation, was observed only in the LC phase of d-C9.5mimPF 6 .

b. $d$-C16mimPF $F_{6}$. The temperature dependence of the low- $Q$ peak in $\mathrm{d}_{-} \mathrm{C} 16 \mathrm{mimPF}_{6}$ was closely studied by the SANS instrument. Figure 5 shows the intensity data around the low- $Q$ peak collected at every $10 \mathrm{~K}$. The width of the low- $Q$ peaks jumped between $390 \mathrm{~K}$ and $400 \mathrm{~K}\left(T_{\mathrm{c}}=394 \mathrm{~K}\right)$, while the peak position was nearly continuous at $T_{\mathrm{c}}$ and shifted to the higher $Q$ side with an increase in temperature.

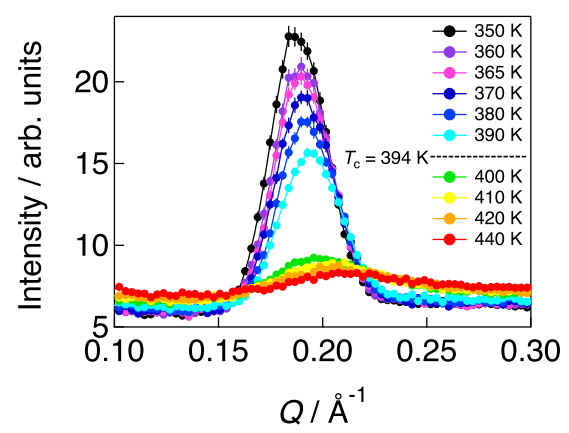

FIG. 5. Neutron diffraction patterns in the low- $Q$ range at around $T_{\mathrm{c}}$ for $\mathrm{d}$ $\mathrm{C}_{16} \mathrm{mimPF}_{6}$ obtained by SANS. Error bars throughout this article represent \pm one standard deviation. 


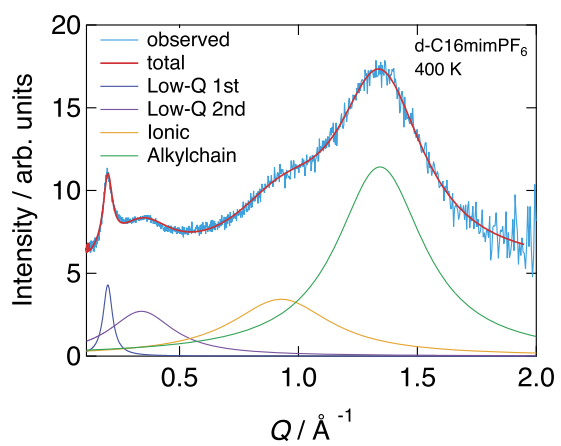

FIG. 6. Result of the fitting using Eq. (1) against the neutron diffraction pattern at $400 \mathrm{~K}$ for $\mathrm{d}-\mathrm{C} 16 \mathrm{mimPF}_{6}$ obtained by TAIKAN. See the text for details.

To make a quantitative analysis on the temperature dependence of the TAIKAN data, the diffraction patterns $I(Q)$ were fitted to the following function:

$$
\begin{aligned}
I(Q)= & \frac{I_{\text {low }}}{\pi} \frac{\Gamma_{\text {low }}}{\left(Q-Q_{\text {low }}\right)^{2}+\Gamma_{\text {low }}^{2}}+\frac{I_{2 \text { nd }}}{\pi} \frac{\Gamma_{2 \text { nd }}}{\left(Q-Q_{2 \text { nd }}\right)^{2}+\Gamma_{2 \text { nd }}^{2}} \\
& +\frac{I_{\text {ion }}}{\pi} \frac{\Gamma_{\text {ion }}}{\left(Q-Q_{\text {ion }}\right)^{2}+\Gamma_{\text {ion }}^{2}}+\frac{I_{\text {alkyl }}}{\pi} \frac{\Gamma_{\text {alkyl }}}{\left(Q-Q_{\text {alkyl }}\right)^{2}+\Gamma_{\text {alkyl }}^{2}} \\
& +(B . G .),
\end{aligned}
$$

where $I_{\mathrm{x}}$ is the integrated intensity, $Q_{\mathrm{x}}$ is the peak position, $\Gamma_{\mathrm{x}}$ is the half-width at half-maximum (HWHM) of the peak, and B.G. is the background with a slope. The subscripts $\mathrm{x}$ (low, 2nd, ion, and alkyl) correspond to the correlations shown in Fig. 1. The representative result of the fitting (d-C16mimPF 6 at $400 \mathrm{~K}$ ) is shown in Fig. 6 . All of the diffraction data for the LC and liquid phases were fitted well.

The parameters obtained by the fitting for d-C $16 \mathrm{mimPF}_{6}$ are shown in Fig. 7(a) for the low- $Q$ peak and Fig. 7(b) for the ionic and alkyl peaks as functions of temperature. For the low- $Q$ peak, our SANS data and previous small-angle x-ray scattering (SAXS) data ${ }^{36}$ are also plotted. All of the data sets agree with each other. The peak position $Q_{\text {low }}$, corresponding to the layer distance of the nanostructure, was continuous at $T_{\mathrm{c}}$ implying the similarity of the local structure between the LC and liquid phases. This continuity for the low- $Q$ peak was observed also in the previous $\mathrm{x}$-ray diffraction data on C16mim[OTf] (trifluoromethanesulfonate) ${ }^{35}$ and
$\mathrm{CnmimBF}_{4}(n=12,14) .{ }^{38}$ The peak position shifted to the high $Q$ side on heating, apparently showing the negative thermal expansion. We guess that this phenomenon is due to the fact that the alkylchains become interdigitated or orientationally disordered, leading to the shorter layer spacing at higher temperatures. The integrated intensity $I_{\text {low }}$ is also continuous, indicating that the number of pairs contributing to the pair correlation functions is unchanged at $T_{\mathrm{c}}$. The peak width $\Gamma$ (HWHM) exhibits an abrupt increase at $T_{\mathrm{c}}$, which will be discussed later (Sec. III A 3).

The peak positions and the integrated intensities of the ionic and alkylchain correlations were continuous at $T_{\mathrm{c}}$ as shown in Fig. 7(b). The peak positions of both correlations shifted to the lower $Q$ side on heating implying that the thermal expansion of inter-ionic and inter-alkylchain lengths does not show any anomaly. The HWHM of the alkylchain correlation was also continuous, whereas that of the ionic correlation exhibited a small jump at $T_{\mathrm{c}}$. This suggests that the ionic correlation becomes stronger in the LC phase than in the liquid phase. The difference between the LC and liquid phases for the ionic and alkylchain correlations is smaller than that for the low- $Q$ peak correlation.

The above results are consistent with our earlier thermal analysis and X-ray diffraction work ${ }^{39}$ and suggest that the nanostructure in the liquid phase of ILs is essentially the same as the layer structure in the $\mathrm{LC}$ phase.

c. $d$-C8mimPF $F_{6}$. Figure 8 (a) shows the temperature dependence of the parameters obtained by the fitting for the low- $Q$ peak of d-C $8 \mathrm{mimPF}_{6}$. This sample did not exhibit the LC-L transition. The hypothetical $T_{\mathrm{c}}$ of d-C $8 \mathrm{mimPF}_{6}$ is about $190 \mathrm{~K}$ as expected from Fig. 2, but the molecular motion is frozen in because the glass transition takes place at $T_{\mathrm{g}}=202 \mathrm{~K}$. The $Q_{\text {low }}$ vs. $T$ curve in Fig. 8 (a) had a kink at $T_{\mathrm{g}} ; Q_{\text {low }}$ slightly decreased on heating below $T_{\mathrm{g}}$, whereas $Q_{\text {low }}$ increased above $T_{\mathrm{g}}$. This tendency is in good agreement with the previous results. $8,9,12,13,15,18$ These data suggest that the interdigitation of alkylchains is promoted and/or the orientation of alkylchains becomes more random on heating above $T_{\mathrm{g}}$, whilst the normal thermal expansion of solids occurs below $T_{\mathrm{g}}$ where the motion of the alkylchains is frozen in. The $\Gamma_{\text {low }}$ curve in Fig. 8(a) also shows a kink at $T_{\mathrm{g}}$ and $\Gamma_{\text {low }}$ is almost unchanged below $T_{\mathrm{g}}$. Above $T_{\mathrm{g}}$, the correlation length of the layer
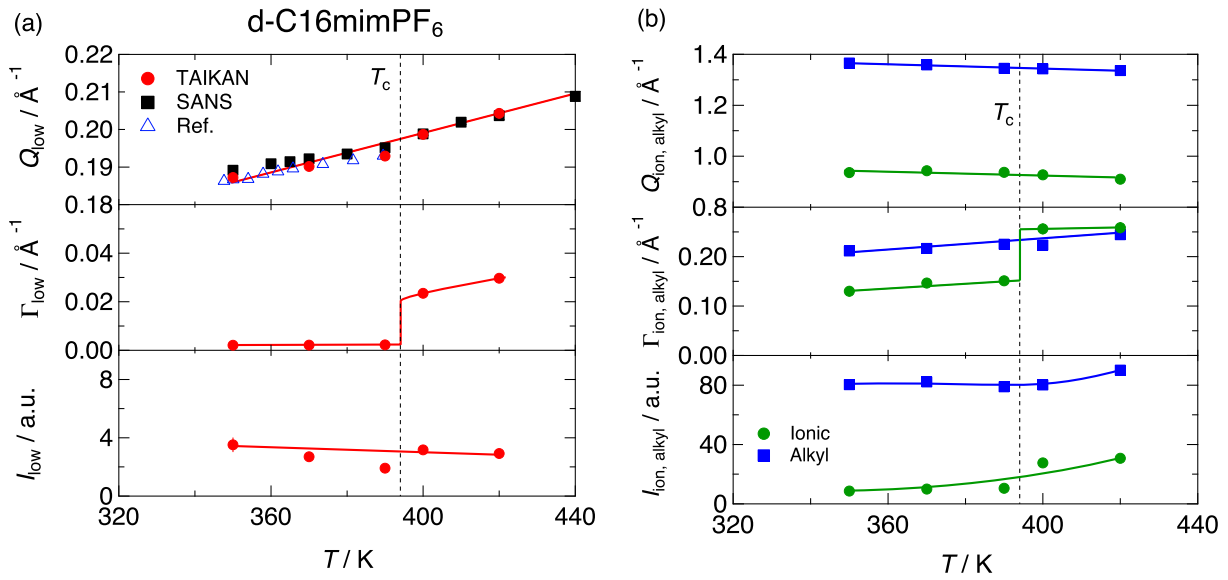

FIG. 7. Temperature dependences of the peak position $Q$, width $\Gamma$, and integrated intensity $I$ for (a) the low- $Q$ peak (red circles and black squares) and (b) the ionic correlation peak (green circles) and alkylchain correlation peak (blue squares) for d-C16mimPF 6 . The literature SAXS data ${ }^{36}$ are also plotted. The vertical dashed lines represent $T_{\mathrm{c}}=394 \mathrm{~K}$. The solid lines are guides to the eye. 


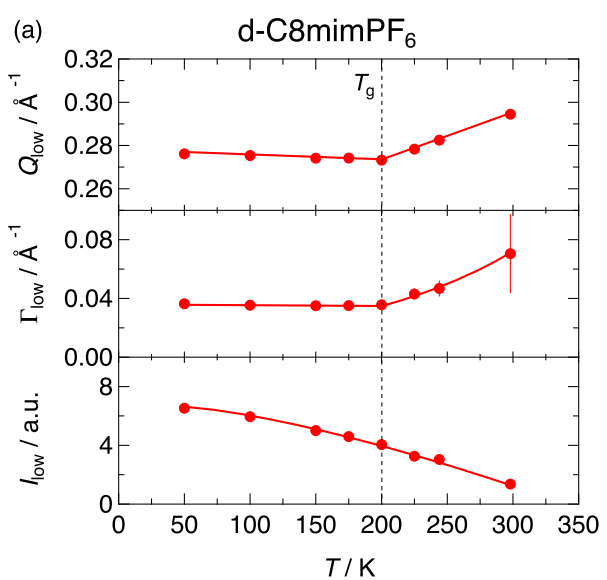

(b)

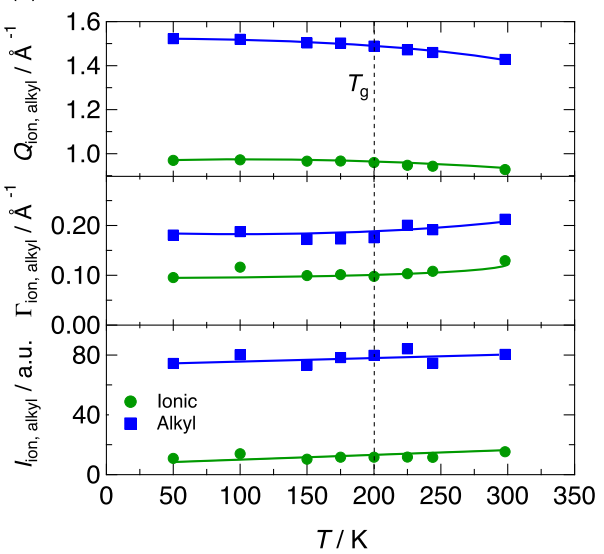

FIG. 8. Temperature dependences of the peak position $Q$, width $\Gamma$, and integrated intensity $I$ for (a) the low- $Q$ peak (red circles) and (b) the ionic correlation peak (green circles) and alkylchain correlation peak (blue squares) for d-C8mimPF ${ }_{6}$. The vertical dashed lines represent $T_{\mathrm{g}}=200 \mathrm{~K}$. The solid lines are guides to the eye. structure decreased along with an increase in temperature. The intensity $I$ continuously increased with a decrease in temperature over the $T_{\mathrm{g}}$ as reported by Kofu et al. ${ }^{15}$ This is interesting in the sense that the structure changes below the freezing temperature $T_{\mathrm{g}}$. There are two different explanations for this phenomenon. One is that the alkylchains are not frozen and orientationally ordered, developing the layer structure, even below $T_{\mathrm{g}}$. It is to be noted that the development of the layer structure is enhanced much below the hypothetical transition temperature $190 \mathrm{~K}$ from the thermodynamic point of view. Another explanation is that the low- $Q$ peak broadens in an energy axis due to the highly fluctuating motions of the alkylchains and the motion becomes sluggish at lower temperatures. To make further discussion, inelastic neutron scattering data are required in this $Q$ region.

The temperature dependence of the peak parameters for the ionic and alkylchain correlations is shown in Fig. 8(b). All parameters were continuous over $T_{\mathrm{g}}$. The peak positions of both correlations shifted to the lower $Q$ side on heating, implying that the inter-ionic and the inter-alkylchain distances exhibit normal thermal expansions independent of the glass transition.

d. $d$-C9.5mimPF . Figures 9(a) and 9(b) show the temperature dependence of the parameters for the low- $Q$ peak and those for the ionic and alkylchain correlation peaks, respectively. At $T_{\mathrm{c}}, \Gamma_{\text {low }}$ and $\Gamma_{\text {ion }}$ exhibit clear jumps, whereas other peak parameters are continuous. Although the L-LC transition of $\mathrm{d}-\mathrm{C} 9.5 \mathrm{mimPF}_{6}$ takes place in a supercooled state, its peak parameters behave as those of $\mathrm{d}-\mathrm{C}_{16} \mathrm{mimPF}_{6}$ at $T_{\mathrm{c}}$. On the other hand, the variation of the peak parameters at $T_{\mathrm{g}}$ is quite similar to those of $\mathrm{d}-\mathrm{C} 8 \mathrm{mimPF}_{6}$ at $T_{\mathrm{g}}$. It is noteworthy that the glass transition of $\mathrm{d}-\mathrm{C} 9.5 \mathrm{mimPF}_{6}$ in the LC phase is

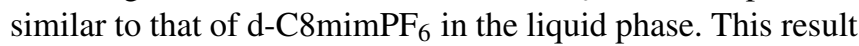
indicates that the disorder, which is frozen at $T_{\mathrm{g}}$, is common for both the LC and liquid phases.

\section{Mechanism of the LC-liquid transition}

The temperature dependence of the correlation length $\xi$ of the layer structure for $\mathrm{d}-\mathrm{C} 9.5 \mathrm{mimPF}_{6}$ and $\mathrm{d}-\mathrm{C} 16 \mathrm{mimPF}_{6}$ is shown in Fig. 10, where $\xi$ is estimated from the relation $\xi$ $=2 \pi / \Gamma$. It is noteworthy that, even in the liquid phases, the correlation length is more than $200 \AA$ corresponding to (6-7) layers with a spacing of ca. $30 \AA$. The correlation grows up in the liquid phase on cooling. At $T_{\mathrm{c}}, \xi$ exhibits a clear jump indicating that this phase transition is of first order. These results indicate that the L-LC transition is due to the abrupt change of the layer structure from short-range order to long-range order. For $\mathrm{d}-\mathrm{C} 9.5 \mathrm{mimPF}_{6}$, the $\xi$ value of the $\mathrm{LC}$ phase is still grown on cooling even below $T_{c}$ and becomes almost constant below $T_{g}$. This is due to the freezing of ionic motions at $T_{g}$ as observed in $\mathrm{d}-\mathrm{C} 8 \mathrm{mimPF}_{6}$.

The LC phase of ILs is similar to a smectic A (SmA) phase of molecular liquid crystals. However, ILs have no nematic (N) (a)

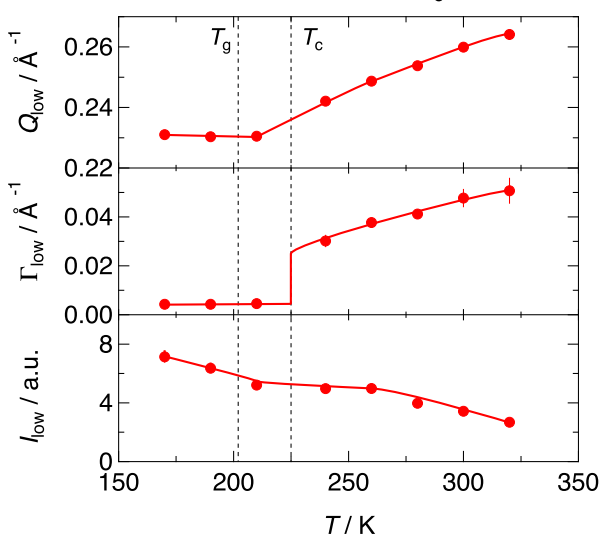

(b)

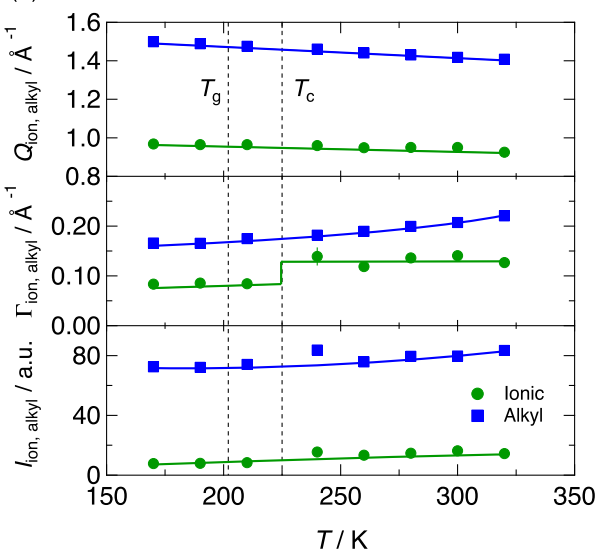

FIG. 9. Temperature dependences of the peak position $Q$, width $\Gamma$, and integrated intensity $I$ for (a) the low- $Q$ peak (red circles) and (b) the ionic correlation peak (green circles) and alkylchain correlation peak (blue squares) for d-C9.5 mimPF $_{6}$. The vertical dashed lines represent $T_{\mathrm{g}}=202 \mathrm{~K}$ and $T_{\mathrm{c}}=225 \mathrm{~K}$. The solid lines are guides to the eye. 


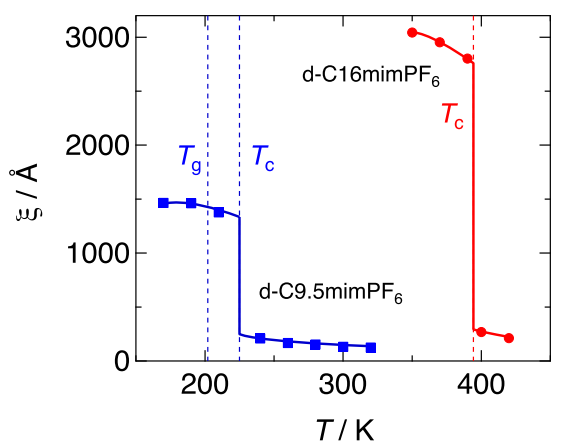

FIG. 10. Temperature dependence of the correlation length $\xi$ for d-C16 $\mathrm{mimPF}_{6}$ (red circles) and d-C9.5 $\mathrm{mimPF}_{6}$ (blue squares). The error bars are smaller than the symbols. The vertical dashed lines represent $T_{\mathrm{c}}=394 \mathrm{~K}$ for d-C16mimPF 6 (blue) and $T_{\mathrm{g}}=202 \mathrm{~K}$ and $T_{\mathrm{c}}=225 \mathrm{~K}$ for d-C9.5mimPF 6 (blue). The solid lines are guides to the eye.

phase, which is orientationally ordered but positionally disordered. In the theory on the transition between the isotropic liquid and SmA phases, ${ }^{88}$ both orientational and the translational order parameters are considered and it is interpreted that the isotropic liquid phase transfers to the SmA LC phase directly at $T_{\mathrm{c}}$ if the translational ordering takes place before or simultaneously with the orientational ordering on cooling. It should be noted that $\mathrm{LC}$ molecules with $\mathrm{N}$ phases possess mesogenic (e.g., phenyl) groups and/or functional groups with dipole interaction, which align molecules unidirectionally, while IL molecules have neither of them. In ILs, the ionic parts aggregate to form the local layer structure, but the structure of ionic parts is not ordered even in the LC phase as indicated by the broad peak of ionic correlation. The alkylchain parts are also fluctuating with high mobility. Thus the orientation of IL molecules is highly disordered even in the LC phase compared with the conventional LC molecules with $\mathrm{N}$ phases. The MD simulations by Saielli group ${ }^{40-45}$ also demonstrated that both alkylchains and imidazolium rings are highly disordered in the SmA LC phase and the orientational and translational order parameters of the SmA LC phases in imidazolium-based ILs are smaller than those in molecular LCs. ${ }^{89}$ The poor orientational order in the LC phase corresponds to the small transition entropies of the $\mathrm{LC}-\mathrm{L}$ phase transition $\left(0.67 \mathrm{~J} \mathrm{~mol}^{-1} \mathrm{~K}\right.$ for d-C9.5 $\mathrm{mimPF}_{6}$ and $2.0 \mathrm{~J} \mathrm{~mol}^{-1} \mathrm{~K}$ for d-C16 $\mathrm{mimPF}_{6}$, respectively). ${ }^{39}$ This situation gives rise to close free energy curves of the $\mathrm{LC}$ and $\mathrm{L}$ phases, producing strong structural fluctuation of the layer structure in the L phase especially in the super-cooled state. $^{39}$

\section{B. Quasielastic neutron scattering}

\section{Coherent QENS: Relaxation of nanostructure}

Figure 11 shows a representative QENS spectrum of d$\mathrm{C}_{16 \mathrm{mimPF}_{6}}$ in the liquid phase observed at $424 \mathrm{~K}$ by DNA with the low-resolution mode: (a) is for the entire spectrum and (b) is for the low intensity part. The data were taken at $Q=0.2 \AA^{-1}\left(\approx Q_{\text {low }}\right)$. Here, the coherent scattering, reflecting the interlayer motion, was mainly observed since the $\sigma_{\text {coh }}$ is larger than the $\sigma_{\text {inc }}$, in the deuterated sample. The QENS spectra were fitted to a conventional function given by

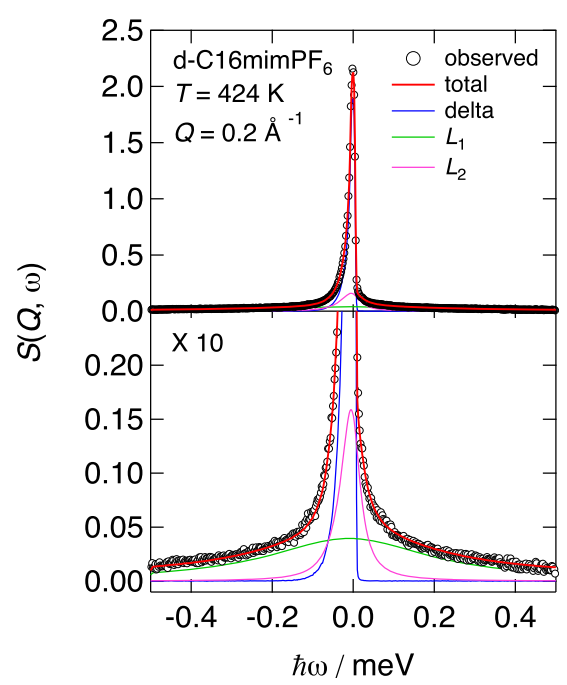

FIG. 11. QENS profiles of d-C16mimPF 6 obtained with the low-resolution mode of DNA at $Q=0.2 \AA^{-1}$ and $T=424 \mathrm{~K}$. Solid curves represent the results of the fitting (see the text for details). (a) is for the entire spectrum and (b) is for the low intensity part.

$$
\begin{aligned}
S(Q, \omega) & =R(Q, \omega)\left\{A_{0} \delta(\omega)+A_{1} L_{1}+A_{2} L_{2}\right\}+(a \omega+b), \\
L_{i}(Q, \omega) & =\frac{1}{\pi} \frac{\Gamma_{i}(Q)}{\left\{(\hbar \omega)^{2}+\Gamma_{i}(Q)^{2}\right\}}, i=1,2,
\end{aligned}
$$

where $R(Q, \omega)$ is the spectrometer resolution function, $\otimes$ is the convolution operator, $\delta$ is the delta function, $L_{\mathrm{i}}$ is the Lorentz functions, $A_{0}, A_{1}$, and $A_{2}$ are the fraction of each term, and $(a \omega+b)$ is the background. $\Gamma_{i}(Q)$ is the half width at half maximum (HWHM) of the Lorentz function. All of the fittings were satisfactory as shown in Fig. 11, indicating that there are two relatively fast relaxations and slow motions which are regarded to be rigid, corresponding to a $\delta$ function, in the present energy resolution. The relaxation times, which are calculated by $\tau=1 / \Gamma$, are labeled $\tau_{1}$ and $\tau_{2}\left(\tau_{1}\right.$ is shorter than $\tau_{2}$ ).

Figure 12 shows the normalized intermediate scattering functions $I(Q, t) / I(Q, 0)$ of d-C16mimPF 6 observed by NSE at $Q=0.2 \AA^{-1}$. Faster relaxations were observed around $0.001 \mathrm{~ns}-0.1 \mathrm{~ns}$ and a slower relaxation around $1 \mathrm{~ns}-100 \mathrm{~ns}$.

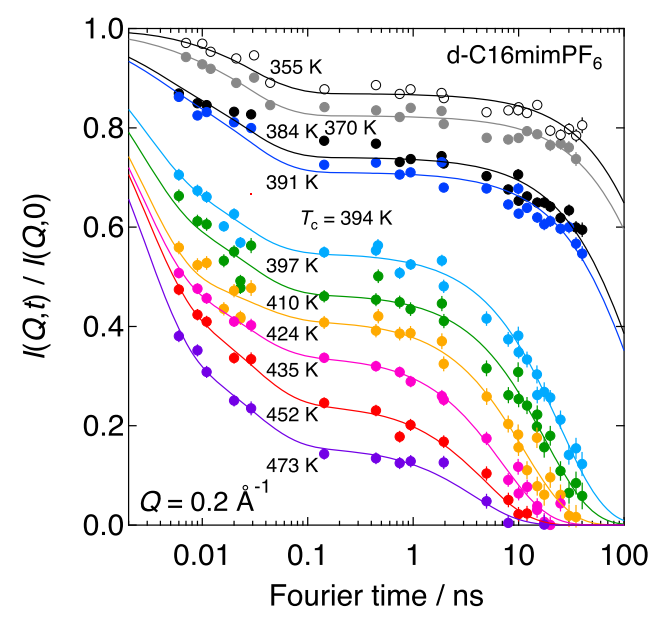

FIG. 12. Intermediate scattering functions of $\mathrm{d}-\mathrm{C} 16 \mathrm{mimPF}_{6}$ observed by NSE at $Q=0.2 \AA^{-1}$. Solid curves represent the results of the fitting (see the text for details). 
(a)

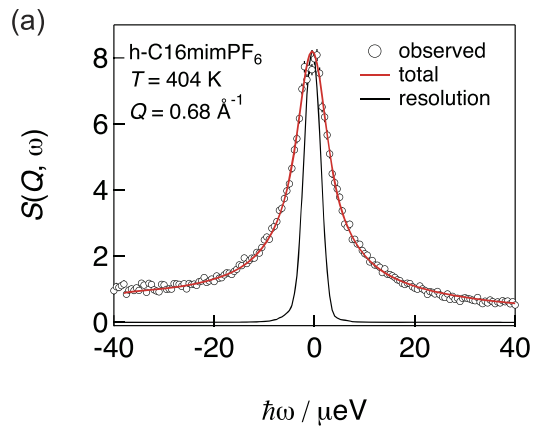

(b)

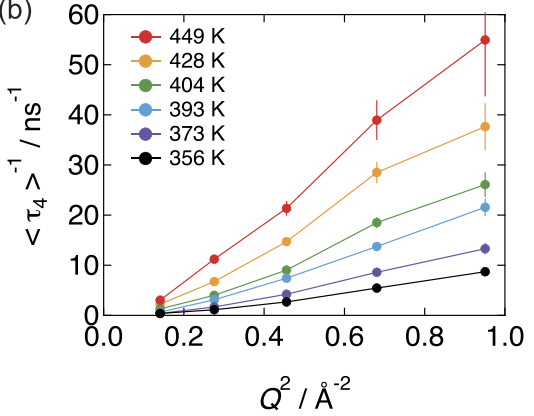

FIG. 13. (a) QENS profiles of $\mathrm{h}$ $\mathrm{C} \mathrm{mimPF}_{6}$ obtained with the highresolution mode of DNA at $Q=0.68 \AA^{-1}$ and $T=404 \mathrm{~K}$. Solid curves represent the results of the fitting (see the text for details). (b) $Q$-dependence of $\left\langle\tau_{4}\right\rangle^{-1}$ of h-C $16 \mathrm{mimPF}_{6}$ obtained by the fitting.
The gap in the relaxation intensity is due to the drastic change in the intensity of the low- $Q$ peaks at $T_{\mathrm{c}}$ (see Fig. 5). These curves were fitted to

$$
\frac{I(Q, t)}{I(Q, 0)}=A_{1} \exp \left(-\frac{t}{\tau_{1}}\right)+A_{2} \exp \left(-\frac{t}{\tau_{2}}\right)+A_{3} \exp \left(-\frac{t}{\tau_{3}}\right)
$$

where $A_{i}$ is the fraction of each relaxation with a constraint of $A_{1}+A_{2}+A_{3}=1$ and $\tau_{i}$ is the relaxation time. The values of $\tau_{1}$ and $\tau_{2}$ were fixed to the values determined by DNA because the DNA data are more reliable in the time region $t<0.1 \mathrm{~ns}$. The relaxation with $\tau_{3}$ corresponds to the slow relaxation which was regarded as a delta function in the analysis of DNA (see Fig. 11). This result is consistent with the results for $\mathrm{d}-\mathrm{C} 8 \mathrm{mimPF}_{6}$ observed by NSE. ${ }^{15}$ In the previous result, the slower $\left(\tau_{3}\right)$ and faster $\left(\tau_{1}\right.$ and $\left.\tau_{2}\right)$ relaxations were assigned to the relaxations of the nanostructure and its broken part, respectively. Actually, the fraction of the broken part increased with increasing temperature. It should be noted that the use of the Debye model for the relaxations with $\tau_{1}, \tau_{2}$, and $\tau_{3}$ is not absolutely right but the simplest approximation to obtain reasonable parameters from the fitting. The relaxation modes could be non-Debye and/or superposition of multiple relaxations.

\section{Incoherent QENS: Ionic diffusion and alkylchain relaxation}

Figure 13(a) shows, as representative data, the $S(Q, \omega)$ spectrum of liquid h-C16mimPF 6 at $T=404 \mathrm{~K}$ and $Q$ $=0.68 \AA^{-1}$. These data were collected by DNA with the highresolution mode. The QENS data were fitted to the Fourier transform of the KWW (Kohlrausch-Williams-Watts) function, ${ }^{90,91}$

$$
S(Q, \omega)=A_{4} R(Q, \omega) F T\left[\exp \left\{-\left(\frac{t}{\tau_{4}}\right)^{\beta}\right\}\right]+(a \omega+b)
$$

where $A_{4}$ is the intensity, $R(Q, \omega)$ is the spectrometer resolution function, $\otimes$ is the convolution operator, $\tau_{4}$ is the relaxation time, $\beta$ is the non-exponential parameter, and $(a \omega+b)$ is the background. The fitting was satisfactory as shown in Fig. 13(a). The obtained $\beta$ values were $0.4-0.5$. The average relaxation times $\left\langle\tau_{4}\right\rangle$ were calculated using a well-known relation $\left\langle\tau_{4}\right\rangle$ $=\tau_{4} \Gamma(1 / \beta) / \beta$, where $\Gamma(\mathrm{x})$ is a gamma function. The relation between $\left\langle\tau_{4}\right\rangle^{-1}$ and $Q^{2}$ is given in Fig. 13(b). $\left\langle\tau_{4}\right\rangle^{-1}$ is nearly proportional to $Q^{2}$ as predicted by Fick's law, implying that this motion is a sort of translational diffusion. This diffusional motion should be the self-diffusion of cations since the $\sigma_{\text {inc }}$ of a proton is much larger than the $\sigma_{\text {coh }}$ of a proton and other atoms. The present result is consistent with the previous incoherent QENS measurements. 8,52-54,56,57,59,61,62

The QENS spectrum of h-C16mimPF 6 in the liquid phase (404 K), which was measured with the low-resolution mode at DNA, is shown in Fig. 14(a). As performed in the high resolution data shown in Fig. 13(a), the data were fitted to the following function:

$$
\begin{aligned}
S(Q, \omega)= & R(Q, \omega)\left\{A_{4} \mathrm{FT}\left[\exp \left\{-\left(\frac{t}{\tau_{4}}\right)^{\beta}\right\}\right]+A_{5} L_{5}+A_{6} L_{6}\right\} \\
& +(a \omega+b), \\
L_{i}(Q, \omega)= & \frac{1}{\pi} \frac{\Gamma_{i}(Q)}{\left\{(\hbar \omega)^{2}+\Gamma_{i}(Q)^{2}\right\}}, i=5,6 .
\end{aligned}
$$

In the present function, two Lorentz functions are added to Eq. (5) as faster relaxation terms. The relaxation times are calculated by $\tau_{i}=1 / \Gamma_{i}$. The values of $\tau_{4}$ and $\beta$ were fixed to those determined by the high-resolution mode. The relaxation times for Lorentz functions are labeled $\tau_{5}$ and $\tau_{6}\left(\tau_{5}<\tau_{6}\right)$. Figure 14(b) shows the $\tau^{-1}$ values versus $Q$ at $356 \mathrm{~K}$ and $404 \mathrm{~K}$. The values are almost independent of $Q$ implying that the faster two terms are of local motions.

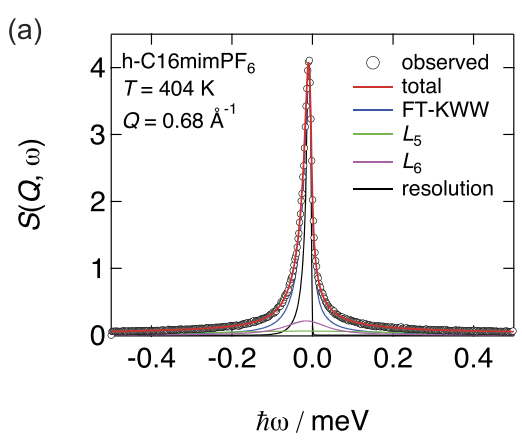

FIG. 14. (a) QENS profiles of h-C16mimPF 6 obtained with the low-resolution mode of DNA at $Q=$ $0.68 \AA^{-1}$ and $T=404 \mathrm{~K}$. Solid curves represent the results of the fitting (see the text for details). (b) $Q$-dependence of $\tau_{5}{ }^{-1}$ and $\tau_{6}{ }^{-1}$ of h-C16mimPF 6 obtained by the fitting. 
Figure 15 shows the Arrhenius plots of $\left\langle\tau_{4}\right\rangle, \tau_{5}$, and $\tau_{6}$ obtained by the incoherent QENS measurement at $Q=0.75 \AA^{-1}$. All of the relaxation times are continuous at $T_{\mathrm{c}}$. The activation energies $\Delta E_{\mathrm{a}}$ for the relaxations with $\left\langle\tau_{4}\right\rangle$, $\tau_{5}$, and $\tau_{6}$ are estimated to be $(29.2 \pm 1.0) \mathrm{kJ} \mathrm{mol}^{-1},(6.1 \pm 1.0)$ $\mathrm{kJ} \mathrm{mol}^{-1}$, and $(9.6 \pm 1.0) \mathrm{kJ} \mathrm{mol}^{-1}$, respectively. The $\Delta E_{\mathrm{a}}$ of the ionic diffusion of $\mathrm{C} \mathrm{mimPF}_{6}$ are $(38.1 \pm 1.2) \mathrm{kJ} \mathrm{mol}^{-1}$, and those of alkylchains are (5-10) $\mathrm{kJ} \mathrm{mol}^{-1}$, not depending much on alkyl lengths and anions. ${ }^{62}$ The present $\Delta E_{\mathrm{a}}$ 's are comparable with the values for $\mathrm{C}_{4} \mathrm{mimPF}_{6}$ and other ionic liquids studied so far.

\section{Overall feature of dynamics observed in $\mathrm{C16mimPF}_{6}$}

Figure 16 shows the temperature dependence of the relaxation times (Arrhenius plots) for the whole relaxations investigated in this work. The $Q$ value of $0.2 \AA^{-1}$ was selected in common since the NSE data were obtained only at this $Q$ value. There are basically 4 relaxation processes.

The slowest mode $\tau_{3}$ exhibits a jump at $T_{\mathrm{c}} ; \tau_{3}$ in the LC phase is about 10 times longer than that in the liquid phase. The activation energies $\Delta E_{\mathrm{a}}$ were calculated to be $(43.3 \pm 9.4)$ $\mathrm{kJ} \mathrm{mol}^{-1}$ in the LC phase and $(41.0 \pm 3.4) \mathrm{kJ} \mathrm{mol}^{-1}$ in the liquid phase. These values are the same as each other within the error range and also essentially the same as $\Delta E_{\mathrm{a}}=(40.4 \pm$ 7.7) $\mathrm{kJ} \mathrm{mol}^{-1}$ of the nanostructure relaxation in $\mathrm{d}-\mathrm{C} 8 \mathrm{mimPF}_{6}$ which has half the length of alkylchains. ${ }^{15}$ On the other hand, the relaxation time of the nanostructure of $\mathrm{d}-\mathrm{C} 8 \mathrm{mimCl}$, which has a smaller anion, is much larger than that of $\mathrm{d}-\mathrm{C} 8 \mathrm{mimPF}_{6} \cdot{ }^{13}$ Thus, the present and previous results indicate that the relaxation of the nanostructure is dominated mainly by the ionic interaction.

Our structural data (Figs. 7 and 9) indicate that ionic correlation becomes slightly stronger at the LC phase accompanied with the ordering of the layer structure. We guess that $\Delta E_{\mathrm{a}}$ in the LC phase is also slightly larger than that in the liquid phase if $\tau_{3}$ is determined more precisely using a NSE spectrometer with a higher energy resolution. It should be effective if the inter- and intra-layer dynamics and furthermore vertical and lateral dynamics are separately measured using the oriented samples. Anyhow, the present work has demonstrated

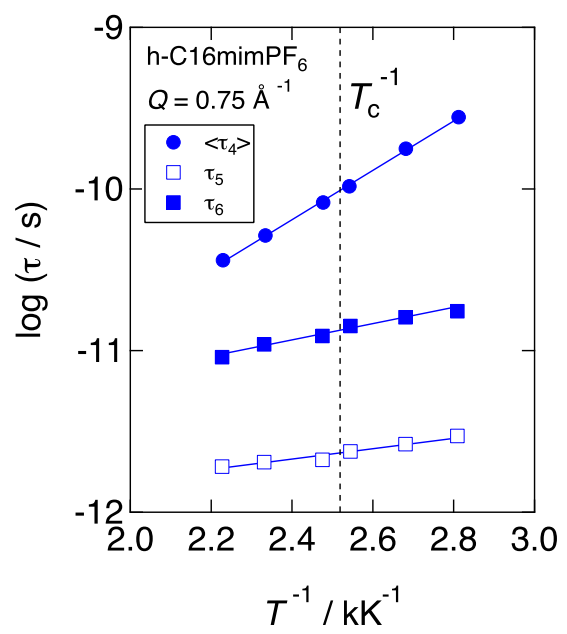

FIG. 15. Arrhenius plots for the relaxation times of $\mathrm{h}-\mathrm{C}_{16} \mathrm{mimPF}_{6}$ at $Q=$ $0.75 \AA^{-1}$. The error bars are smaller than the symbols.

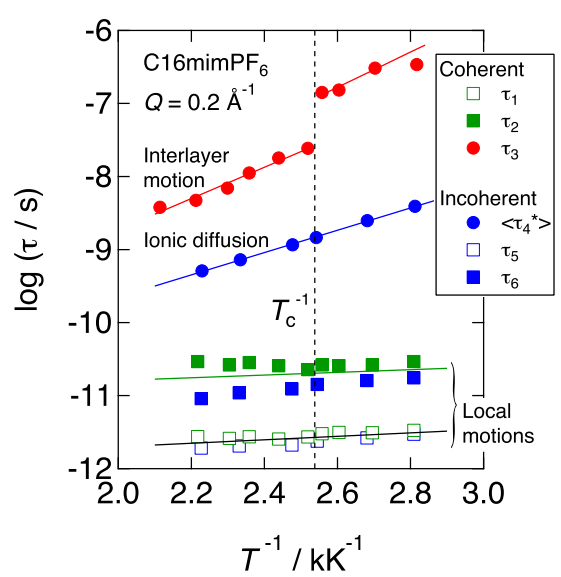

FIG. 16. Overall Arrhenius plots for the relaxation times of d-C16mimPF and h-C $16 \mathrm{mimPF}_{6}$ at $Q=0.2 \AA^{-1}$. The error bars are smaller than the symbols.

the relaxation of the nanostructure in both the LC and liquid phases of ILs for the first time.

The relaxation times of ionic diffusion $\left\langle\tau_{4}{ }^{*}\right\rangle$ at $Q=0.2 \AA^{-1}$ were estimated from $\left\langle\tau_{4}\right\rangle$ at $Q=0.75 \AA^{-1}$ by assuming $\left\langle\tau_{4}\right\rangle^{-1}=D Q^{2}$, where $D$ is the diffusion constant. As mentioned in Fig. 15 (at $\left.Q=0.75 \AA^{-1}\right),\left\langle\tau_{4}{ }^{*}\right\rangle$ was also almost continuous at $T_{\mathrm{c}}$. This is consistent with the fact that the change in the ionic correlation at $T_{\mathrm{c}}$ is quite small. The ionic interaction is important for the formation and dynamics of the nanostructure, as mentioned before, but its change at $T_{\mathrm{c}}$ is small and a drastic change appeared only at a smaller $Q$ (in the layer structure and dynamics). This is an essential feature of the LC-liquid transition of $\mathrm{C}_{16 \mathrm{mimPF}}$.

The relaxation times $\tau_{5}$ and $\tau_{6}$ at $Q=0.2 \AA^{-1}$ are assumed to be the same values at $Q=0.75 \AA^{-1}$ because these relaxations are of local motions as indicated in Fig. 14(b). We guess that the relaxations with $\tau_{1}$ and $\tau_{5}$ are identical relaxations associated with intra-ionic motions although the former is observed in coherent QENS, while the latter is observed in the incoherent one. There may be a similar situation for the relaxations with $\tau_{2}$ and $\tau_{6}$. The temperature dependence of these relaxation times is quite small. This may be because the relaxational motions consist of many local hindered rotations around the $\mathrm{C}-\mathrm{C}$ bonds in the alkylchain and those of the imidazolium rings. In this situation, the temperature dependence of each relaxational mode is smeared owing to the superposition effect.

As described above, the relaxation times, except $\tau_{3}$, for the interlayer motion, were continuous at $T_{\mathrm{c}}$. This result is consistent with the structural data demonstrating the continuity for the ionic and alkylchain correlations at $T_{\mathrm{c}}$. This is also consistent with the previous QENS work for h-C16mimPF 6 observing the ionic diffusion and alkylchain relaxations. ${ }^{36}$ Their EISF (elastic incoherent structure factor) curve has no discontinuity at $T_{\mathrm{c}}$. The discontinuity of $\tau_{3}$ is similar to the dynamical change at SmA-isotropic liquid transitions in conventional liquid crystals. For the conventional systems, the interlayer diffusion investigated by NMR measurement ${ }^{92}$ and MD simulations ${ }^{93}$ exhibits a jump at the transitions and the activation energy in the LC phase is higher than that in the

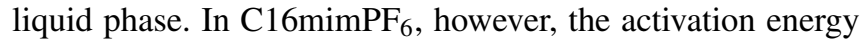


of the LC phase is close to that of the liquid phase within the error of the present experiment. This is a characteristic feature of ILs with a relatively disordered structure in the LC phase and a relatively ordered structure in the liquid phase.

\section{CONCLUSION}

The ND experiment has revealed that the layer structure exists in both the SmA LC and liquid phases. These two phases are distinguished by the correlation length $\xi$ of the layer structure; $\xi$ of the LC phase is about ten times longer than that in the liquid phase. The ionic part (imidazolium rings and anions) is slightly ordered in the LC phase, while the alkylchains are almost disordered in both the $\mathrm{LC}$ and liquid phases. These results are obtained for both d-C16mimPF 6 and d-C9.5 $\mathrm{mimPF}_{6}$, suggesting the universal feature independent of the alkylchain length. The structural difference between the LC and liquid phases in ILs is much smaller than that in the conventional LCs (e.g., cyanobiphenyl LCs). This is consistent with the difference in the transition entropies; $\Delta S$ (IL) $<\Delta S$ (cyanobiphenyl LCs). ${ }^{39}$

The QENS experiment for $\mathrm{C}_{16} \mathrm{mimPF}_{6}$ has demonstrated that the dynamical change at $T_{\mathrm{c}}$ (LC-liquid) is quite consistent with the structural one. The ionic and alkylchain relaxations are almost continuous at $T_{\mathrm{c}}$. On the other hand, the relaxation time of the layer nanostructure is ten times longer in the LC phase than that in the liquid phase.

Thus, the LC and L phases of ILs are quite similar in both the structure and dynamics, except those related to the layer structure which is the most characteristic feature of ILs.

\section{ACKNOWLEDGMENTS}

The travel expense for the NSE experiment at NCNR, NIST (USA) was supported by General User Program for Neutron Scattering Experiments, Institute for Solid State Physics, The University of Tokyo (Proposal No. 14611). Access to the NGB-30m-SANS and the NSE spectrometer was provided by the Center for High Resolution Neutron Scattering, a partnership between the National Institute of Standards and Technology and the National Science Foundation under Agreement No. DMR-1508249. The experiments in MLF, J-PARC were performed with the approval of J-PARC (Proposal Nos. 2014A0303 for TAIKAN and 2014A0156 and 2015A0262 for DNA). M.N. acknowledges funding support of cooperative Agreement No. 70NANB15H259 from NIST, U.S. Department of Commerce.

Certain trade names and company products are identified in order to specify adequately the experimental procedure. In no case does such identification imply recommendation or endorsement by the National Institute of Standards and Technology, nor does it imply that the products are necessarily the best for the purpose.

\footnotetext{
${ }^{1}$ M. J. Earle and K. R. Seddon, Pure Appl. Chem. 72, 1391-1398 (2000).

${ }^{2}$ M. Armand, F. Endres, D. R. MacFarlane, H. Ohno, and B. Scrosati, Nat. Mater. 8, 621-629 (2009).

${ }^{3}$ A. E. Somers, P. C. Howlett, D. R. MacFarlane, and M. Forsyth, Lubricants 1, 3-21 (2013).

${ }^{4}$ R. Hayes, G. G. Warr, and R. Atkin, Chem. Rev. 115, 6357-6426 (2015).
}

${ }^{5}$ J. C. Araque, J. J. Hettige, and C. J. Margulis, J. Phys. Chem. B 119, $12717-$ 12740 (2015).

${ }^{6}$ O. Russina and A. Triolo, Exp. Methods Phys. Sci. 49, 213-278 (2017).

${ }^{7}$ C. Hardacre, J. D. Holbrey, S. E. J. McMath, D. T. Bowron, and A. K. Soper, J. Chem. Phys. 118, 273-278 (2003).

${ }^{8}$ A. Triolo, A. Mandanici, O. Russina, V. Rodrigues-Mora, M. Cutroni, C. Hardacre, M. Nieuwenhuyzen, H.-J. Bleif, L. Keller, and M. A. Ramos, J. Phys. Chem. B 110, 21357-21364 (2006).

${ }_{9}^{9}$ A. Triolo, O. Russina, H.-J. Bleif, and E. Di Cola, J. Phys. Chem. B 111, 4641-4644 (2007).

${ }^{10}$ A. Triolo, O. Russina, B. Fazio, R. Triolo, and E. Di Cola, Chem. Phys. Lett. 457, 362-365 (2008).

${ }^{11}$ C. Hardacre, J. D. Holbrey, C. L. Mullan, T. G. A. Youngs, and D. T. Bowron, J. Chem. Phys. 133, 074510 (2010).

${ }^{12}$ B. Aoun, A. Goldbach, M. A. González, S. Kohara, D. L. Price, and M.-L. Saboungi, J. Chem. Phys. 134, 104509 (2011).

${ }^{13}$ O. Yamamuro, T. Yamada, M. Kofu, M. Nakakoshi, and M. Nagao, J. Chem. Phys. 135, 054508 (2011).

${ }^{14}$ O. Russina and A. Triolo, Faraday Discuss. 154, 97-109 (2012).

${ }^{15}$ M. Kofu, M. Nagao, T. Ueki, Y. Kitazawa, Y. Nakamura, S. Sawamura, M. Watanabe, and O. Yamamuro, J. Phys. Chem. B 117, 2773-2781 (2013).

${ }^{16}$ M. A. A. Rocha, C. M. S. S. Neves, M. G. Freire, O. Russina, A. Triolo, J. A. P. Coutinho, and L. M. N. B. F. Santos, J. Phys. Chem. B 117, 1088910897 (2013).

${ }^{17}$ K. Fujii, S. Seki, K. Ohara, Y. Kameda, H. Doi, S. Saito, and Y. Umebayashi, J. Solution Chem. 43, 1655-1668 (2014).

${ }^{18}$ D. Pontoni, J. Haddad, M. Di Michiel, and M. Deutsch, Soft Matter 13, 6947-6955 (2017).

${ }^{19}$ O. Russina, F. Lo Celso, N. V. Plechkova, and A. Triolo, J. Phys. Chem. Lett. 8, 1197-1204 (2017).

${ }^{20}$ H. Weiss, J. Mars, H. Li, G. Kircher, O. Ivanova, A. Feoktystov, O. Soltwedel, M. Bier, M. Mezger, J. Phys. Chem. B 121, 620-629 (2017).

${ }^{21}$ C. P. Cabry, L. D' Andrea, K. Shimizu, I. Grillo, P. Li, S. Rogers, D. W. Bruce, J. N. C. Lopes, and J. M. Slattery, Faraday Discuss. 206, 265-289 (2018).

${ }^{22}$ Y. Wang and G. A. Voth, J. Am. Chem. Soc. 127, 12192-12193 (2005).

${ }^{23}$ J. N. C. Lopes, M. F. Costa Gomes, and A. A. H. Pádua, J. Phys. Chem. B 110, 16816-16818 (2006).

${ }^{24}$ D. Bedrov, O. Borodin, Z. Li, and G. D. Smith, J. Phys. Chem. B 114, 4984-4997 (2010).

${ }^{25}$ H. V. R. Annapureddy, H. K. Kashyap, P. M. De Biase, and C. J. Margulis, J. Phys. Chem. B 114, 16838-16846 (2010).

${ }^{26}$ K. Shimizu, C. E. S. Bernardes, and J. N. C. Lopes, J. Phys. Chem. B 118, 567-576 (2014).

${ }^{27}$ K. Shimizu and J. N. C. Lopes, J. Mol. Liq. 210, 257-263 (2015).

${ }^{28}$ M. Sha, Y. Liu, H. Dong, F. Luo, F. Jiang, Z. Tang, G. Zhu, and G. Wu, Soft Matter 12, 8942-8949 (2016).

${ }^{29}$ A. Yethiraj, J. Phys.: Condens. Matter 28, 414020 (2016).

${ }^{30}$ E. C. Wu, H. J. Kim, and L. A. Peteanu, J. Phys. Chem. B 121, 1100-1107 (2017).

${ }^{31}$ C. J. Bowlas, D. W. Bruce, and K. R. Seddon, Chem. Commun. 1996, $1625-1626$.

${ }^{32}$ C. M. Gordon, J. D. Holbrey, A. R. Kennedy, and K. R. Seddon, J. Mater. Chem. 8, 2627-2636 (1998).

${ }^{33}$ J. D. Holbrey and K. R. Seddon, J. Chem. Soc., Dalton Trans. 1999, $2133-$ 2140.

${ }^{34}$ C. K. Lee, H. W. Huang, and I. J. B. Lin, Chem. Commun. 2000, 1911-1912.

${ }^{35}$ A. E. Bradley, C. Hardacre, J. D. Holbrey, S. Johnston, S. E. J. McMath, and M. Nieuwenhuyzen, Chem. Mater. 14, 629-635 (2002).

${ }^{36}$ J. De Roche, C. M. Gordon, C. T. Imrie, M. D. Ingram, A. R. Kennedy, F. Lo Celso, and A. Triolo, Chem. Mater. 15, 3089-3097 (2003).

${ }^{37}$ F. Xu, K. Matsumoto, and R. Hagiwara, Dalton Trans. 41, 3494-3502 (2012).

${ }^{38}$ Y. Nozaki, K. Yamaguchi, K. Tomida, N. Taniguchi, H. Hara, Y. Takikawa, K. Sadakane, K. Nakamura, T. Konishi, and K. Fukao, J. Phys. Chem. B 120, 5291-5300 (2016).

${ }^{39}$ F. Nemoto, M. Kofu, and O. Yamamuro, J. Phys. Chem. B 119, 5028-5034 (2015).

${ }^{40}$ G. Saielli, Soft Matter 8, 10279-10287 (2012).

${ }^{41}$ G. Saielli, G. A. Voth, and Y. Wang, Soft Matter 9, 5716-5725 (2013).

${ }^{42}$ Y. Ji, R. Shi, Y. Wang, and G. Saielli, J. Phys. Chem. B 117, 1104-1109 (2013).

${ }^{43}$ G. Saielli, A. Bagno, and Y. Wang, J. Phys. Chem. B 119, 3829-3836 (2015).

${ }^{44}$ G. Saielli, J. Phys. Chem. B 120, 2569-2577 (2016).

${ }^{45}$ G. Saielli and Y. Wang, J. Phys. Chem. B 120, 9152-9160 (2016). 
${ }^{46}$ A. Noda, K. Hayamizu, and M. Watanabe, J. Phys. Chem. B 105, 4603-4610 (2001).

${ }^{47}$ H. Tokuda, K. Hayamizu, K. Ishii, M. A. B. H. Susan, and M. Watanabe, J. Phys. Chem. B 108, 16593-16600 (2004).

${ }^{48}$ J. H. Antony, A. Dölle, D. Mertens, P. Wasserscheid, W. R. Carper, and P. G. Wahlbeck, J. Phys. Chem. A 109, 6676-6687 (2005).

${ }^{49}$ K. Nakamura and T. Shikata, ChemPhysChem 11, 285-294 (2010).

${ }^{50}$ J. R. Sangoro, C. Iacob, S. Naumov, R. Valiullin, H. Rexhausen, J. Hunger, R. Buchner, V. Strehmel, J. Kärger, and F. Kremer, Soft Matter 7, 1678-1681 (2011).

${ }^{51}$ T. Endo, S. Widgeon, P. Yu, S. Sen, and K. Nishikawa, Phys. Rev. B 85, 054307 (2012)

${ }^{52}$ A. Triolo, O. Russina, V. Arrighi, F. Juranyi, S. Janssen, and C. M. Gordon, J. Chem. Phys. 119, 8549-8557 (2003).

${ }^{53}$ A. Triolo, O. Russina, C. Hardacre, M. Nieuwenhuyzen, M. A. Gonzalez, and H. Grimm, J. Phys. Chem. B 109, 22061-22066 (2005).

${ }^{54}$ Y. Inamura, O. Yamamuro, S. Hayashi, H. Hamaguchi, Phys. B 385-386, 732-734 (2006).

${ }^{55}$ O. Russina, M. Beiner, C. Pappas, M. Russina, V. Arrighi, T. Unruh, C. L. Mullan, C. Hardacre, and A. Triolo, J. Phys. Chem. B 113, 8469-8474 (2009).

${ }^{56}$ B. Aoun, M. A. González, J. Ollivier, M. Russina, Z. Izaola, D. L. Price, and M.-L. Saboungi, J. Phys. Chem. Lett. 1, 2503-2507 (2010).

${ }^{57}$ S. M. Chathoth, E. Mamontov, S. Dai, X. Wang, P. F. Fulvio, and D. J. Wesolowski, Europhys. Lett. 97, 066004 (2012).

${ }^{58}$ T. Yamaguchi, K. Mikawa, S. Koda, K. Fujii, H. Endo, M. Shibayama, H. Hamano, and Y. Umebayashi, J. Chem. Phys. 137, 104511 (2012).

${ }^{59}$ B. Aoun, M. A. González, M. Russina, D. L. Price, and M.-L. Saboungi, J. Phys. Soc. Jpn. 82, SA002 (2013).

${ }^{60}$ J. L. Bañuelos, G. Feng, P. F. Fulvio, S. Li, G. Rother, N. Arend, A. Faraone, S. Dai, P. T. Cummings, and D. J. Wesolowski, Carbon 78, 415-427 (2014).

${ }^{61}$ D. L. Price, O. Borodin, M. A. González, M. Kofu, K. Shibata, T. Yamada, O. Yamamuro, and M.-L. Saboungi, J. Phys. Chem. Lett. 8, 715-719 (2017).

${ }^{62} \mathrm{M}$. Kofu, M. Tyagi, Y. Inamura, K. Miyazaki, and O. Yamamuro, J. Chem. Phys. 143, 234502 (2015).

${ }^{63}$ K. Fujii, M. Shibayama, T. Yamaguchi, K. Yoshida, T. Yamaguchi, S. Seki, H. Uchiyama, A. Q. R. Baron, and Y. Umebayashi, J. Chem. Phys. 138, 151101 (2013).

${ }^{64}$ K. R. Seddon, A. Stark, and M.-J. Torres, in Clean Solvents: Alternative Media for Chemical Reactions and Processing, ACS Symposium Series (American Chemical Society, 2002), Chap. 4.

${ }^{65}$ H. Tokuda, S. Tsuzuki, M. A. B. H. Susan, K. Hayamizu, and M. Watanabe, J. Phys. Chem. B 110, 19593-19600 (2006).

${ }^{66}$ M. Fukuda, M. Terazima, and Y. Kimura, J. Chem. Phys. 128, 114508 (2008).

${ }^{67}$ W. Makino, R. Kishikawa, M. Mizoshiri, S. Takeda, and M. Yao, J. Chem. Phys. 129, 104510 (2008).

${ }^{68}$ A. Šantić, W. Wrobel, M. Mutke, R. D. Banhatti, and K. Funke, Phys. Chem. Chem. Phys. 11, 5930-5394 (2009).
${ }^{69}$ T. Yamaguchi, S. Miyake, and S. Koda, J. Phys. Chem. B 114, 8126-8133 (2010).

${ }^{70}$ T. Yamaguchi, E. Nakahara, and S. Koda, J. Phys. Chem. B 118, 5752-5759 (2014).

${ }^{71}$ T. Cosby, Z. Vicars, Y. Wang, and J. Sangoro, J. Phys. Chem. Lett. 8, 35443548 (2017).

${ }^{72}$ C. Daguenet, P. G. Dyson, I. Krossing, A. Oleinikova, J. Slattery, C. Wakai, and H. Weingartner, J. Phys. Chem. B 110, 12682-12688 (2006).

${ }^{73}$ K. Yamamoto, M. Tani, and M. Hangyo, J. Phys. Chem. B 111, 4854-4858 (2007).

${ }^{74}$ A. Stoppa, J. Hunger, R. Buchner, G. Hefter, A. Thoman, and H. Helm, J. Phys. Chem. B 112, 4854-4858 (2008).

${ }^{75}$ J. Hunger, A. Stoppa, S. Schrödle, G. Hefter, and R. Buchner, ChemPhysChem 10, 723-733 (2009).

${ }^{76}$ D. A. Turton, J. Hunger, A. Stoppa, G. Hefter, A. Thoman, M. Walther, R. Buchner, and K. Wynne, J. Am. Chem. Soc. 131, 11140-11146 (2009).

${ }^{77}$ M. Mizoshiri, T. Nagao, Y. Mizoguchi, and M. Yao, J. Chem. Phys. 132, 164510 (2010).

${ }^{78}$ J. A. Widegren, E. M. Saurer, K. N. Marsh, and J. W. Magee, J. Chem. Thermodyn. 37, 569-575 (2005).

${ }^{79}$ J. R. Sangoro, M. Mierzwa, C. Iacob, M. Paluch, and F. Kremer, RSC Adv. 2, 5047-5050 (2012).

${ }^{80}$ O. Zech, A. Stoppa, R. Buchner, and W. Kunz, J. Chem. Eng. Data 55, 1774-1778 (2010).

${ }^{81}$ S. Takata, J. Suzuki, T. Shinohara, T. Oku, T. Tominaga, K. Ohishi, H. Iwase, T. Nakatani, Y. Inamura, T. Ito, K. Suzuya, K. Aizawa, M. Arai, T. Otomo, and M. Sugiyama, JPS Conf. Proc. 8, 036020 (2015).

${ }^{82}$ Y. Inamura, T. Nakatani, J. Suzuki, and T. Otomo, J. Phys. Soc. Jpn. 82, SA031 (2013).

${ }^{83}$ C. J. Glinka, J. G. Barker, B. Hammouda, S. Krueger, J. J. Moyer, and W. J. Orts, J. Appl. Crystallogr. 31, 430-445 (1998).

${ }^{84}$ S. R. Kline, J. Appl. Crystallogr. 39, 895-900 (2006).

${ }^{85}$ K. Shibata, N. Takahashi, Y. Kawakita, M. Matsuura, T. Yamada, T. Tominaga, W. Kambara, M. Kobayashi, Y. Inamura, T. Nakatani, K. Nakajima, and M. Arai, JPS Conf. Proc. 8, 036022 (2015).

${ }^{86}$ R. T. Azuah, L. R. Kneller, Y. Qiu, P. L. W. Tregenna-Piggott, C. M. Brown, J. R. D. Copley, and R. M. Dimeo, J. Res. Natl. Inst. Stand. Technol. 114, 341-358 (2009).

${ }^{87}$ N. Rosov, S. Rathgeber, and M. Monkenbusch, in Scattering From Polymers: Characterization by X-Rays, Neutrons, and Light, ACS Symposium Series (American Chemical Society, 2000), Chap. 7.

${ }^{88}$ P. K. Mukherjee, H. Pleiner, and H. R. Brand, Eur. Phys. J. E 4, 293-297 (2001).

${ }^{89}$ M. F. Palermo, A. Pizzirusso, L. Muccioli, and C. Zannoni, J. Chem. Phys. 138, 204901 (2013).

${ }^{90}$ R. Kohlrausch, Ann. Phys. (Berlin) 167, 56- 82 (1854); F. Kohlrausch, ibid. 195, 337-368 (1863).

${ }^{91}$ G. Williams and D. C. Watts, Trans. Faraday Soc. 66, 80 (1970).

${ }^{92}$ G. J. Krüger, Phys. Rep. 82, 229-269 (1982).

${ }^{93}$ M. A. Bates and G. R. Luckhurst, J. Chem. Phys. 120, 394-403 (2004). 